\title{
EFFECT OF DEPTH ON THE SEISMIC RESPONSE OF SQUARE TUNNELS
}

\author{
Ulas CILINGIR $^{\mathrm{i})}$ and S. P. Gopal MADABhushii
}

\begin{abstract}
Response of tunnels to earthquake induced loads is a complex dynamic soil-structure interaction problem. While there seems to be a general consensus that tunnels in rock perform adequately during earthquake events, the seismic performance of shallow tunnels in soils is less certain. More experimental and field data is needed to better understand the dynamic tunnel-soil interaction. In this paper, the behaviour of relatively shallow tunnels of square cross-section located in a sand deposit is investigated using dynamic centrifuge modelling and complimentary Finite Element analysis. Emphasis is given on the effect of tunnel axis depth on the seismic response of square tunnels. Dynamic centrifuge tests were carried out on model tunnels at different depths of embedment. Accelerations around the tunnel and earth pressures on the linings were measured. Tunnel deformations were also recorded using a fast digital camera. Particle Image Velocimetry (PIV) analyses were conducted to measure soil and lining deformations. Results show that for the cases investigated, the depth of the tunnel does not effect the deformation pattern of the tunnel significantly during an earthquake event; however it affects the amount of amplification of accelerations through the tunnel, the magnitude of dynamic earth pressures and the magnitude of the lining forces.
\end{abstract}

Key words: dynamic problems, soil-structure interaction, tunnels and underground openings (IGC: E12/G8/H5)

\section{INTRODUCTION}

Tunnels are increasingly used in urban environments worldwide and constitute an important part of the civil infrastructure. In seismic regions these structures are under earthquake induced risks. Generally tunnels in rock perform reasonably well during earthquake events. However, some damage to tunnels was recorded during the Kobe Earthquake in Japan (1995), Duzce Earthquake in Turkey (1999), Chi-chi Earthquake in Taiwan (1999), Bam Earthquake in Iran (2003) and lastly the Wenchuan Earthquake in China (2008). Further, seismic performance of relatively shallow tunnels that are located in soil strata is less certain. There are attempts to evaluate the response of tunnels to seismic activity ranging from simplified elastic analysis to more complex numerical models. Hashash et al. (2001) provides an in-depth review of these methods.

A recent field study by Wang et al. (2009) showed that all of the 18 mountain tunnels investigated in Sichuan area suffered several types of damage such as portal failures, rockfalls, tunnel failures due to fault action, which causes shearing off the whole tunnel section and cracking and spalling of concrete liners due to seismic wave action etc. Previous studies also show that tunnels may suffer from slope failures, liquefaction induced floatation or sinking and ovaling or racking deformations due to transverse shear waves (Hashash et al., 2001;
Wang et al., 2001; Pakbaz and Yareevand, 2005). Indeed, ovaling and racking deformations during earthquakes are reported to cause most serious damage to tunnel except for the case of whole tunnel section being sheared by a fault (Penzien, 2000). Reports also show that the type of soil or rock surrounding the tunnel, the intensity of the earthquake, the distance to the earthquake hypocentre, the type of tunnel lining and the maximum ground acceleration and the depth of the tunnel below the ground surface affect the behavior of the tunnels (Hashash et al., 2001). Reported damage appears to decrease with increasing overburden depth. However, there is very little experimental data to verify this.

Recently, Shibayama et al. (2010) conducted centrifuge experiments on horseshoe-shaped tunnel models by applying cyclic shear deformations on the centrifuge models and measured earth pressures and bending moments around the lining. The tunnel models were statically sheared and therefore the findings do not include the effects of mass inertia. However, they have shown that the formation of the arch action is strongly affected by the structural rigidity of the tunnels. They have also provided important experimental data on the effects of fixity of the horseshoe-shaped tunnel lining on the cyclic bending moments the lining experiences.

This paper is focused on the effects of tunnel depth on the seismic behaviour of square tunnels under transverse shear $\left(S_{\mathrm{h}}\right)$ waves and aims to provide experimental and

i) Research Associate, Engineering Department, University of Cambridge, United Kingdom (uc206@cam.ac.uk).

ii) Reader in Geotechnical Engineering, Engineering Department, University of Cambridge, United Kingdom.

The manuscript for this paper was received for review on September 28, 2009; approved on February 7, 2011.

Written discussions on this paper should be submitted before January 1, 2012 to the Japanese Geotechnical Society, 4-38-2, Sengoku, Bunkyoku, Tokyo 112-0011, Japan. Upon request the closing date may be extended one month. 
numerical data by presenting the results of centrifuge tests and complementary Finite Element (FE) simulations. Rectangular and square tunnels are usually constructed by cut \& cover technique and have shallow tunnel axes such that the depth-to-width $(\mathrm{H} / \mathrm{W})$ ratio is about 2 . In this paper, the depth-to-width ratios of 1 and 1.8 were considered for the model tunnels. The results from these models tests were compared with each other to delineate the effects of depth on the seismic behaviour of the tunnels. In order to facilitate a direct comparison of the results, tunnels with similar flexibilities were used. The "flexibility" of the tunnel is defined as the ability of the tunnel structure to deform under shear stresses applied by the surrounding soil without inducing high internal lining stresses. It is the flexural stiffness of the surrounding medium compared to the tunnel and defined by Eq. (1) for square tunnels (Wang, 1993). In Eq. (1), $G_{\mathrm{m}}$ is the shear modulus of the soil, $I$ is the moment of inertia of the lining (per unit width), $E_{1}$ is the Young's modulus of the lining material and $W$ is the width of the tunnel.

$$
F_{\text {square }}=\frac{G_{\mathrm{m}}}{12}\left(\frac{W^{3}}{E_{1} I}\right)
$$

It should be noted that, in a given soil stratum a smaller flexibility ratio would mean that the tunnel is rigid while a larger flexibility ratio would indicate a flexible tunnel. It is possible to encounter flexibility ratios for square cutand-cover tunnels, which range from almost zero up to 30-40 for very flexible tunnels constructed using thin sheet pile walls.

\section{CENTRIFUGE MODELLING}

Geotechnical centrifuge modelling is a well established method commonly used both in research and industry since its introduction in early 20th century. The purpose of the centrifuge modelling is to obtain in-situ stressstrain relationships in small scale models by increasing body forces through application of high centrifugal accelerations. Scaling laws are needed to interpret the results and convert them into prototype scale. Schofield (1981) employed dimensional analysis methods to derive centrifuge scaling laws.

Dynamic centrifuge tests described in this paper are conducted at Cambridge Schofield Centrifuge Centre with $10 \mathrm{~m}$ diameter "Turner" beam centrifuge, which has a capacity of $150 \mathrm{~g}$-tonne. Application of earthquake input motions were possible by devising Stored Angular Momentum (SAM) actuator, which was designed to apply sinusoidal input motions up to a prototype frequency of $5 \mathrm{~Hz}$ (with higher frequency harmonics) and peak ground acceleration of $0.4 \mathrm{~g}$ under a maximum centrifugal acceleration of $100 \mathrm{~g}$. More details on the design and operation of the SAM earthquake actuator are given by Madabhushi et al. (1998).

A total of 4 models, each having different tunnel flexibility and depth, were tested in dry Fraction E silica sand. The details of the test configurations are presented in Table 1 and Fig. 2. All of the tunnels have flexibility ratios greater than unity. Model tunnels UC04 and UC06 have the flexibility ratios of 14 and 20 (relatively rigid) and the model tunnels UC10 and UC11 have the flexibility ratios of 247 and 330 (relatively flexible) as given in Table 1, where the lining thicknesses were also reported. Flexibility ratios were calculated using soil stiffness values which were calculated using Hardin and Drnevich (1972) stress-dependent, small strain stiffness formulation for sand. This formulation was reported to fit reasonably well to soil stiffness Vs strain data obtained for Fraction E sand as reported Ghosh and Madabhushi (2002). Model tunnels were constructed using aluminium alloys with a width of $5 \mathrm{~m}$ at prototype scale $(100 \mathrm{~mm}$ in model scale). Two groups of tunnel models one at $5 \mathrm{~m}(\mathrm{H} / \mathrm{W}=$ $1.0)$ and the other at $9 \mathrm{~m}(\mathrm{H} / \mathrm{W}=1.8)$ below ground surface, were tested. The depth is measured from soil surface to the tunnel axis.

Dry pluviation method was used to pour the sand. Relative density was kept around $45 \%$. Aluminium model container, which has internal dimensions of $500 \mathrm{~mm}$ long by $235 \mathrm{~mm}$ wide and $300 \mathrm{~mm}$ deep, has a perspex viewing window, which makes it possible to record soil and lining deformations inside the box using a fast recording digital camera at 1000 frames per second (see Fig. 1). Fast camera sees the soil and the tunnel through a $45^{\circ}$ mirror attached to the model container. Recorded digital images were analysed using Particle Image Velocimetry (PIV) technique. It involves dividing the image into small patches and finding the location of those patches in consecutive images using cross-correlation functions. More detail on the application of PIV technique to geotechnical modelling is given by White (2002). This study is the first time where PIV technique was employed on high speed camera images obtained at a high resolution during a dynamic centrifuge test.

Table 1. Parameters of the centrifuge models and input motion (prototype scale)

\begin{tabular}{c|c|c|c|c|c|c|c}
\hline Test ID & $\begin{array}{c}\text { Tunnel Depth } \\
(\mathrm{m})\end{array}$ & $\begin{array}{c}\text { Model Depth } \\
(\mathrm{m})\end{array}$ & $\begin{array}{c}\text { Depth to Width ratio } \\
(H / W)\end{array}$ & $\begin{array}{c}\text { Lining Thickness } \\
(\mathrm{m})\end{array}$ & Flexibility Ratio & $\begin{array}{c}\text { Maximum base } \\
\text { acceleration } \\
(\% \mathrm{~g})\end{array}$ & $\begin{array}{c}\text { Duration of the } \\
\text { input motion (s) }\end{array}$ \\
\hline UC04 & 5 & 14 & 1.0 & 0.15 & 14 & 27.0 & 25 \\
\hline UC06 & 9 & 18 & 1.8 & 0.15 & 20 & 24.0 & 25 \\
\hline UC10 & 9 & 18 & 1.8 & 0.06 & 330 & 21.8 & 20 \\
\hline UC11 & 5 & 14 & 1.0 & 0.06 & 247 & 25.0 & 20 \\
\hline
\end{tabular}




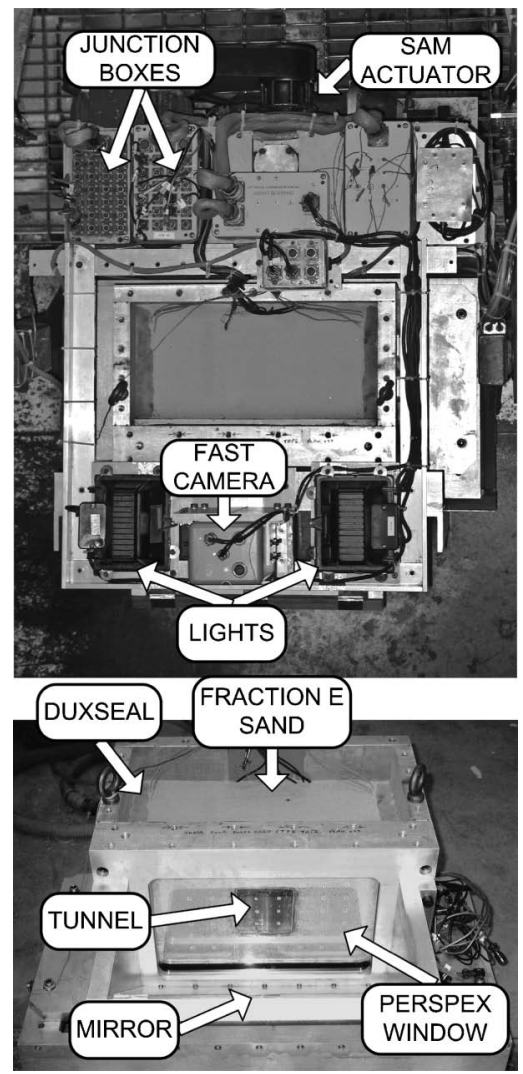

Fig. 1 Centrifuge package and model container

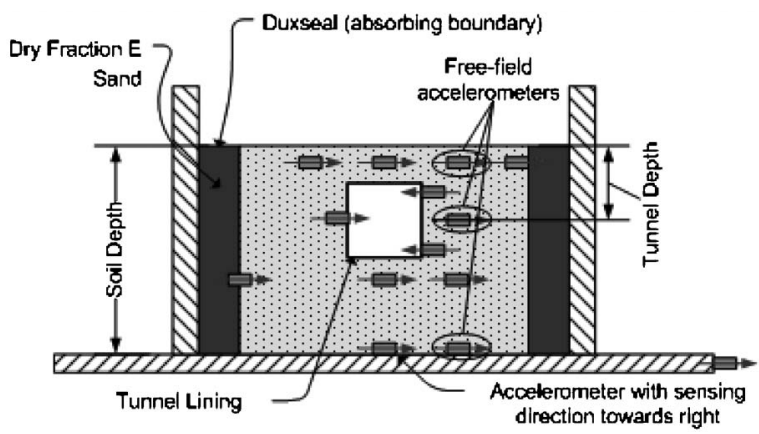

Fig. 2 Schematic of a typical test layout

All of the centrifuge tests were conducted at $50 \mathrm{~g}$ centrifugal acceleration with frequency of the input motion at $50 \mathrm{~Hz}$ in model scale $(1.0 \mathrm{~Hz}$ in prototype). Accelerations around the tunnel were measured with miniature piezoelectric accelerometers. In some of the tests, total earth pressure transducers were fixed on the tunnel lining. Schematic of a typical test layout is given in Fig. 2 .

DUXSEAL was used on the side walls of the rigid container as shown in Fig. 1 in order to reduce boundary effects. It is a putty like plastic material which is reported to being able to isolate up to $65 \%$ of incident $\mathrm{P}$-waves (Steedman and Madabhushi, 1991). Similar results on the effectiveness of DUXSEAL ${ }^{\circledR}$ on isolating free travelling $\mathrm{P}$-waves across the rigid containers were reported by Cheney et al. (1988), Campbell et al. (1991) and Lenke et al. (1991). Figure 3 shows acceleration time histories

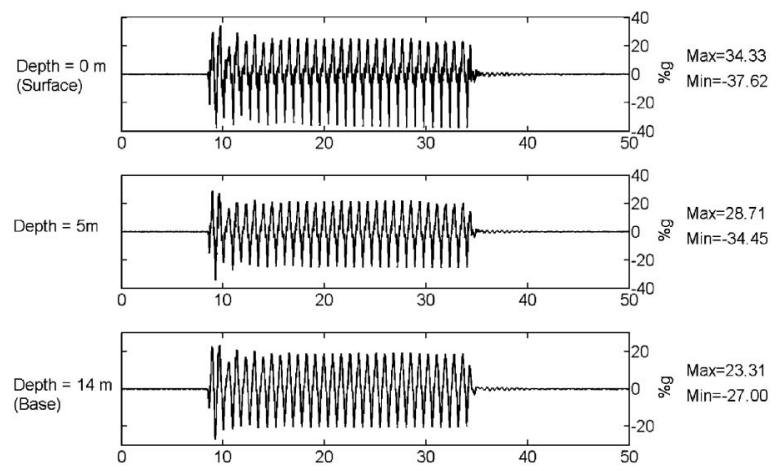

Fig. 3 Free-field accelerations

recorded in the free-field at three levels. It is possible to see that the accelerations are amplified from model base to the soil surface as would be expected for the case of loose to medium dense sand stratum subjected to base shaking tested in a laminar model container or an Equivalent Shear Beam (ESB) model container. This suggests that the boundary effects due to the end wall are not significant and the DUXSEAL is able to absorb the wave reflections adequately.

\section{FINITE ELEMENT MODELLING}

Finite Element (FE) simulations were carried out in order to complement the results of the centrifuge tests. SIMULIA Abaqus v6.7 general purpose finite element software was preferred due to its reliable, bug-free code implementation, contact simulation capabilities, large material model library and its ability to be customized by external FORTRAN subroutines. Implicit Newmark- $\beta$ method was used in the analyses with a modified NewtonRaphson solver algorithm, which Abaqus/Standard module uses by default.

Fraction E sand was modelled by a hardening, nonassociative elasto-plastic Mohr-Coulomb material model. A FORTRAN subroutine was embedded to this model which calculates the elastic stiffness of the soil inside the yield surface at each time increment using stress dependent small strain stiffness formulation given by Hardin and Drnevich (1972). Tunnel linings were modelled with an elastic-perfectly plastic material model. The yield strength of the aluminium linings was taken as $260 \mathrm{MPa}$ (0.2\% proof strength of aluminium alloy BS6082). DUXSEAL ${ }^{\circledR}$ was modelled as an elastic material with 30 $\%$ material damping. Material damping was introduced by Rayleigh damping which is defined in Eq. (2):

$$
\zeta_{\mathrm{i}}=\frac{\alpha_{\mathrm{R}}}{2 \omega_{\mathrm{i}}}+\frac{\beta_{\mathrm{R}} \omega_{\mathrm{i}}}{2}
$$

where $\xi_{\mathrm{i}}$ is the damping ratio at mode $i, \omega_{\mathrm{i}}$ is the circular frequency of the mode $i$ and $\alpha_{\mathrm{R}}$ and $\beta_{\mathrm{R}}$ are Rayleigh damping coefficients proportional to the mass and stiffness respectively. For the analyses described in this paper, no mass-proportional damping was used because massproportional damping simulates a model moving through 
a viscous ether and is not representative for the dry sand model used in the centrifuge tests. The stiffness proportional damping $\beta_{\mathrm{R}}$ was taken as $8.0 \times 10^{-5}$ for the Fraction $\mathrm{E}$ sand. The initial voids ratio was $0.86,0.83,0.82$ and 0.82 for the tests UC04, UC06, UC10 and UC11 respectively. In all the analyses the Poisson's ratio was taken as 0.3 , friction angle was taken as $32^{\circ}$, the dilation angle was taken as $4^{\circ}$ and the cohesion was kept at 0.001 $\mathrm{MPa}$ for the Fraction E sand.

Node-to-node frictional contact was defined between tunnel lining and the sand as well as between DUXSEAL ${ }^{\circledR}$ and the sand. Finite friction formulation available in Abaqus was used which allows Coulomb type frictional shear resistance to be defined on the contact boundaries. Coefficient of friction between the tunnel lining and the sand was calculated using the relationship given by Kishida and Uesugi (1987) between normalised roughness of the lining surface and the coefficient of friction assuming that sand particles do not indent into the aluminium lining. Normalised roughness was measured by performing profilometer tests on tunnel linings.

Eight-node continuum quadrilateral plane-strain elements were used for both soil and the DUXSEAL ${ }^{\circledR}$. 3noded quadratic beam elements in plane with Timoshenko beam formulation were used for the tunnel linings. Structured meshes were used for simulations (see Fig. 4). Meshes were refined around the tunnels up to half the size of the free-field elements. The size of the mesh was decided by considering desired accuracy and computational cost of each analysis. A soil depth to element length ratio of 20 was adopted for the free-field, which resulted in approximately 18 hours of analysis time with an Intel Core 2 Duo personal computer with 4 GB of RAM working under Windows Vista operating system. This element size is approximately 80 times smaller than the wavelength of the first vibration mode and therefore enough to capture high frequency response of the system.

Automatic time incrementation for dynamic problems is available in Abaqus. If the time increment yields stable solutions over certain number of time steps, the time increment is increased by a certain percentage. If it does not, the time increment is decreased automatically by a fraction defined by the controlling parameters. User can define the maximum, minimum and initial time incre-

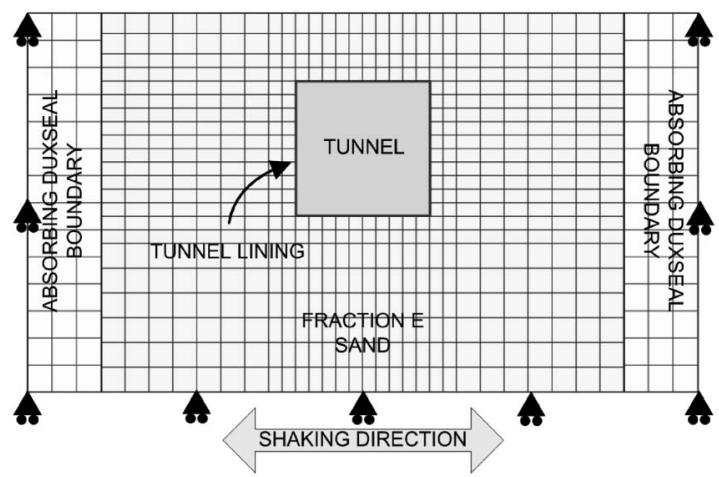

Fig. 4 Typical Finite Element mesh ments. In the analyses described here, a maximum time increment $\Delta t=5 \times 10^{-6} \mathrm{~s}$ was used. It is approximately 20 times smaller than the travel time of the propagating shear wave through a single element of $20 \mathrm{~mm}$ size in model scale as suggested by Haigh et al. (2005). The ratio between the wavelength of the shear waves propagating through the soil and the tunnel width is approximately 11 for the shallow tunnel and 14 for the deep tunnel.

\section{ACCELERATIONS}

Recorded accelerations around the tunnel showed that the amplification of accelerations is affected as the depth of the tunnel changes. Figures 5(a) and (b) show amplifications from bedrock to the top of the tunnel as the frequency changes. They are transfer functions which were calculated by dividing the cross spectral density of an acceleration trace by the power spectral density of the input signal. Low energy frequency components of the input signal were eliminated because these components may introduce artificially high values due to divide-by-zero errors. Coherences between input and output signals were also checked. Frequency components with low coherence were eliminated even if they have sufficient energy to appear in transfer function plots. This method was previously used by Brennan (2003) and Thusyanthan (2005) for evaluating amplification of maximum base accelera-

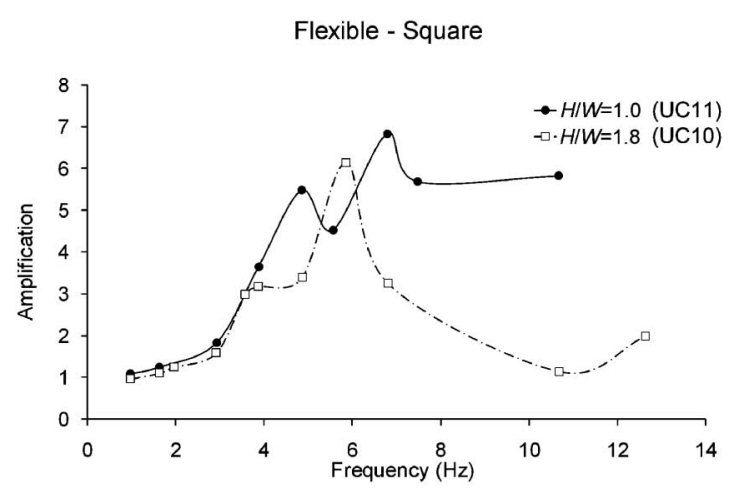

(a)

Rigid - Square

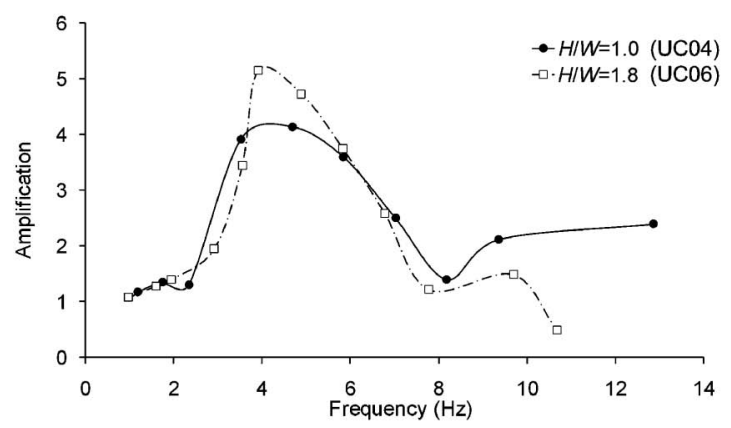

(b)

Fig. 5 Frequency vs. amplification of accelerations from bedrock to the top of the tunnel (a) relatively flexible square tunnels (UC10 and $\mathrm{UC11}$ (b) relatively rigid square tunnels (UC04 and UC06) 


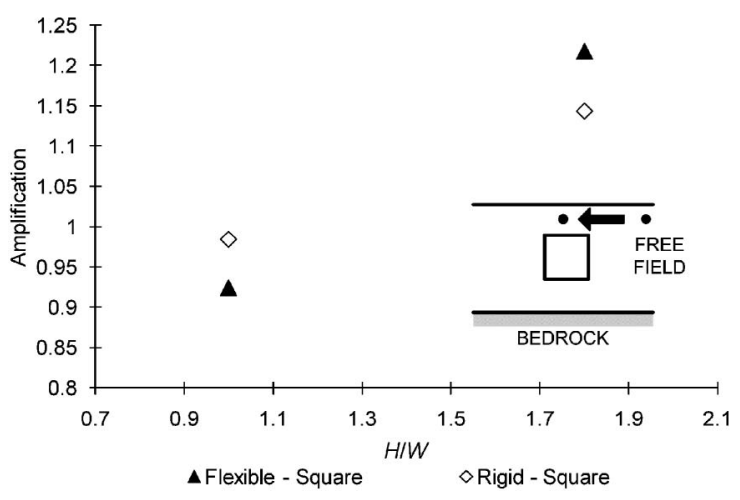

Fig. 6 Amplification of peak acceleration from free-field to the top of the tunnel at different depth-to-width ratios $(\mathrm{H} / \mathrm{W})$

tions in centrifuge models.

Figures 5(a) and (b) show that accelerations above the tunnel show larger amplification for shallow model compared to the deeper model for whole range of frequencies except around $6 \mathrm{~Hz}$. For higher frequencies the difference between amplification values gets larger.

Figure 6 compares the accelerations above the tunnel with free field with increasing depth-to-width ratio. It is possible to see that the peak acceleration above the tunnel gets larger than the acceleration in free-field as depth-towidth ratio increases. This means shear waves travelling upwards are isolated better through shallower tunnels compared to their deep counterparts. This effect is more pronounced for flexible tunnels.

\section{EARTH PRESSURES}

Earth pressures were measured using total earth pressure cells attached to the relatively flexible square tunnel models UC10 and UC11 at various locations around the lining. Figure 7 shows typical earth pressure time histories from two centrifuge tests. The earth pressures given in the figure are incremental (dynamic) earth pressures over the static values. Both of these earth pressure time histories were measured at the same place on the lining. The approximate location of the earth pressure transducer is also shown in the figure. Figure 7(a) shows the input motion and dynamic earth pressure time histories from the test UC06 (relatively rigid, deep tunnel model) and Fig. 7(b) shows the input motion and dynamic earth pressure time histories from the test UC10 (relatively flexible, deep tunnel model). In both of these figures it is possible to see that the earth pressure time history consists of three main zones. First zone is defined by first couple of cycles which causes the earth pressures to reach to a maximum value. It can be defined as a transient stage. A steady-state cyclic zone follows the initial part with a gradual change in average earth pressures towards a final residual value. It was observed that the residual earth pressures build up after each subsequent earthquake as well. These transient, steady state and residual zones in the earth pressure time histories were observed in other
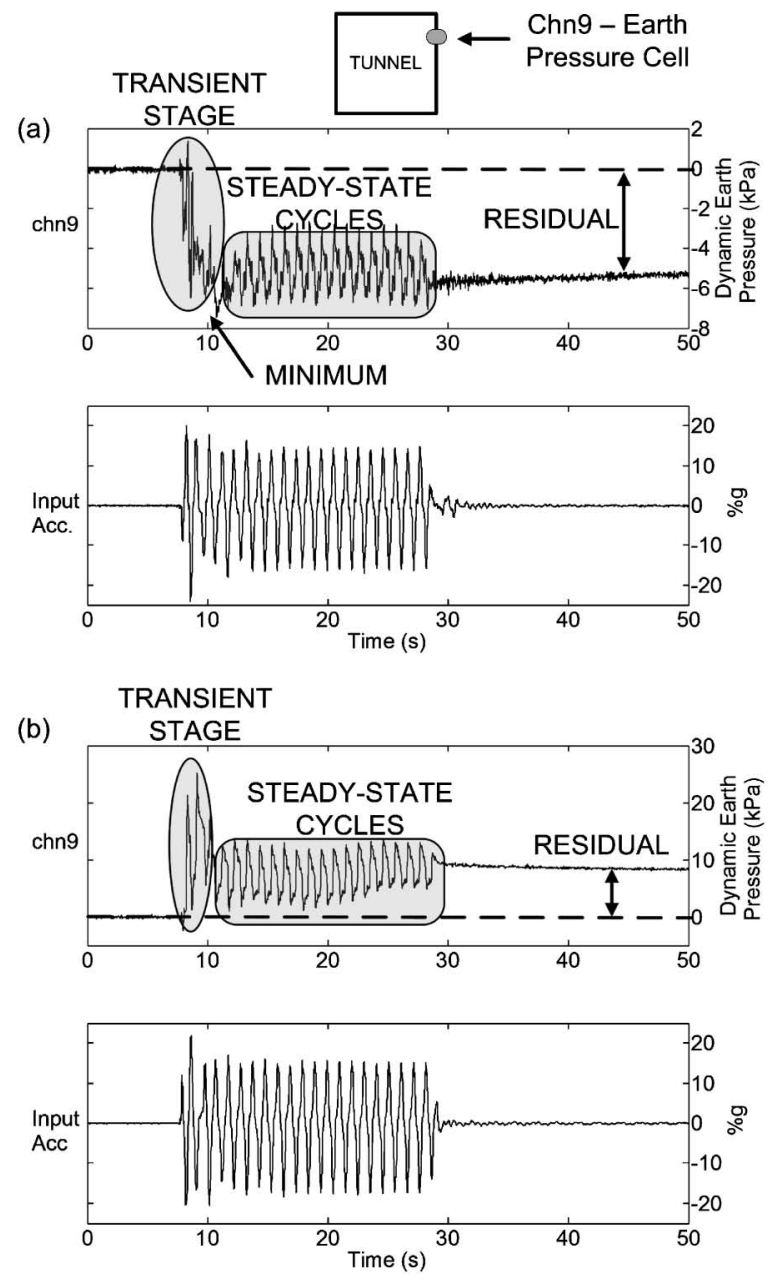

Fig. 7 Typical earth pressure time history from centrifuge tests (a) UC06 and (b) UC10 (prototype scale)

earth pressure measurements from earth pressure transducers located around the tunnel linings as well.

Figure 8 shows the maximum and residual dynamic earth pressures for tunnels at different depths. Letters A, $\mathrm{B}, \mathrm{C}$ and $\mathrm{D}$ indicate the corners of the square tunnels. $\mathrm{X}$ Coordinate starts at point A and follows the letters in anti-clockwise direction. Negative values of the dynamic earth pressures mean that the earth pressures decreased at those points. Solid and dashed lines are the results from FE analyses whereas circular and triangular markers show the measurements from centrifuge tests.

Results from both relatively flexible (UC10 and UC11) and relatively rigid (UC04 and UC06) tunnel models are presented in Fig. 8. Both FE results and centrifuge measurements show that deeper tunnels exhibit larger changes in earth pressure values. That is also true for the residual earth pressures that remain after the end of cyclic loading. Largest changes in earth pressure occur near the corners of tunnels as expected. But they exhibit substantial differences for tunnels of different flexibilities. In relatively flexible tunnel models, active earth pressure changes are observed near the corners $\mathrm{A}$ and $\mathrm{C}$ whereas passive changes occur near corners B and D. Relatively rigid tunnels on the other hand tend to show passive earth 


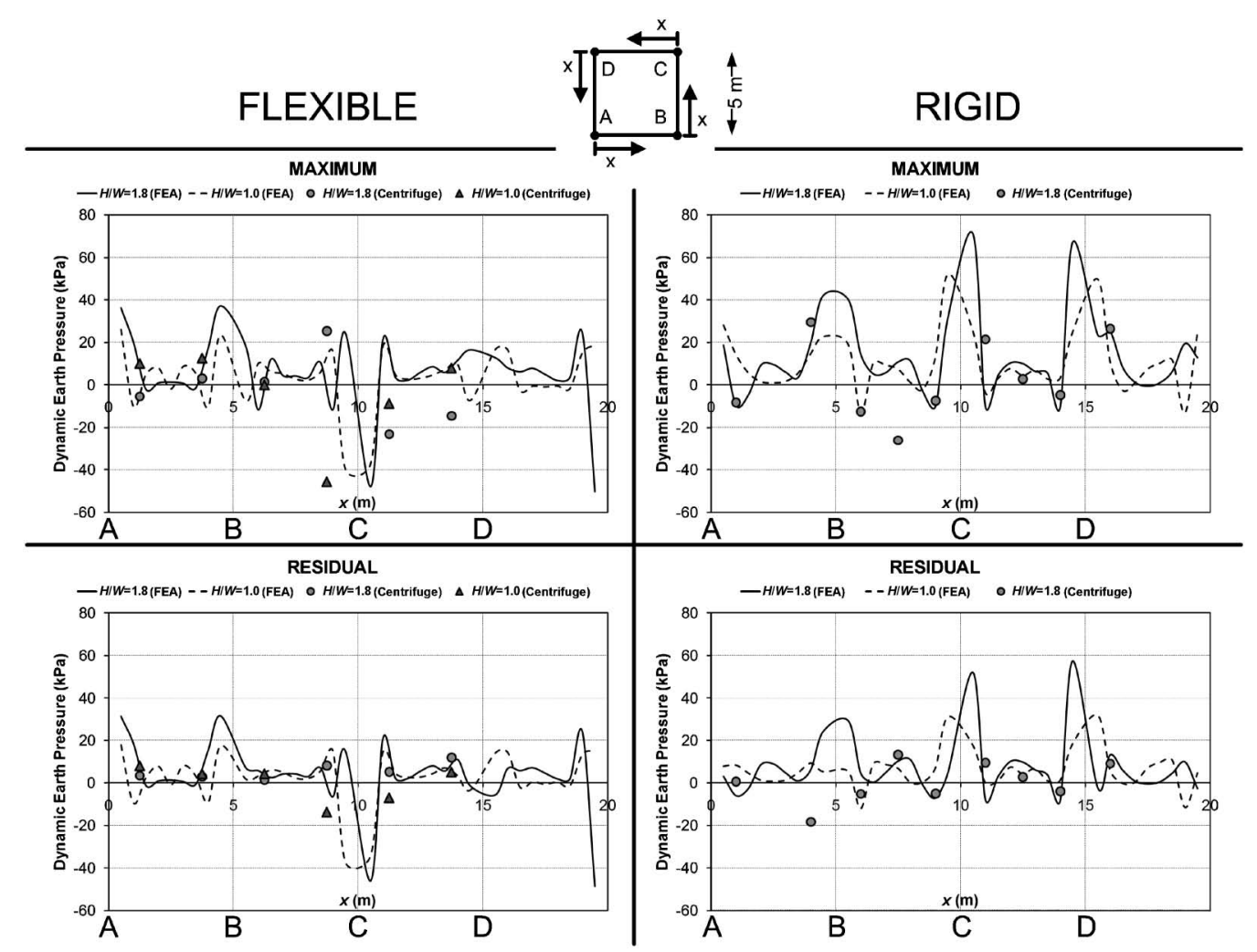

Fig. 8 Maximum and residual earth pressures around square tunnels (prototype scale)

pressure development all around. This can be attributed to different types of deformation mechanism for tunnels of different flexibility. However, the effect of the depth on these deformation mechanisms seems to be small compared to the effects of flexibility itself.

\section{TUNNEL LINING DEFORMATIONS}

Figure 9 shows tunnel lining and soil deformations for deep and shallow, relatively flexible tunnel models UC10 and UC11 respectively from PIV analyses. All the coordinates are in model scale and the arrows, which show the soil deformations around the tunnel from zero to maximum acceleration phase, are magnified by 100 . It is possible to see that the soil deformations are amplified from bottom to top, but no substantial difference between the deep and shallow tunnels could be observed. Both of the tunnel models showed sway deformations. For deeper tunnel model (UC10), crown of the tunnel deforms more than the crown of shallow tunnel model (UC11).

Finite element predictions for cyclic, maximum and residual bending moments are given in Figs. 11 and 12 for relatively flexible and relatively rigid square tunnels respectively. Maximum bending moment is the largest magnitude bending moment ever experienced in that particular location in the lining during the earthquake. The sign convention for the bending moments is given in Fig. 10 according to which caving-in deformations of the lining results in positive bending moments in the middle of the walls. X-coordinate follows the path defined by the corners of the square tunnel, which were marked by letters A,
$\mathrm{B}, \mathrm{C}$ and $\mathrm{D}$ in anti-clockwise direction where corner $\mathrm{A}$ is the bottom-left corner of the tunnel.

Dynamic bending moments for models $\mathrm{UC10}$ and UC11 shown in Fig. 11 reveal that deeper tunnels are subjected to larger maximum cyclic and residual bending moments compared to the shallow tunnels. Maximum cyclic bending moment is defined as the maximum incremental bending moment ever experienced by the lining at the point of interest. Its numerical value is found by subtracting the static bending moment from the maximum dynamic bending moment calculated by the FE analysis at that point. Magnitudes of the maximum and residual bending moments are close to each other and they are always negative near the corners of the tunnel. On the contrary, the sign of the cyclic bending moments alternate near the corners from negative to positive. This shows that first couple of transient-state cycles cause the tunnel walls to deform to reach maximum bending moments and then the tunnel enters a cyclic steady-state period where the tunnel sways at each cycle causing alternating positive and negative bending moments on the corners. At the end of the earthquake, residual bending moments are left in the tunnel lining. The fact that the magnitude and distribution of the residual bending moments are close to the maximum values shows that the first couple of cycles cause accumulation of largest portion of the residual. Subsequent cycles do not contribute to the deformations much. This is in agreement with centrifuge tests results, especially with the measured earth pressure time histories previously shown in Fig. 7. It is possible to see that depth does not affect this behaviour 
UC10

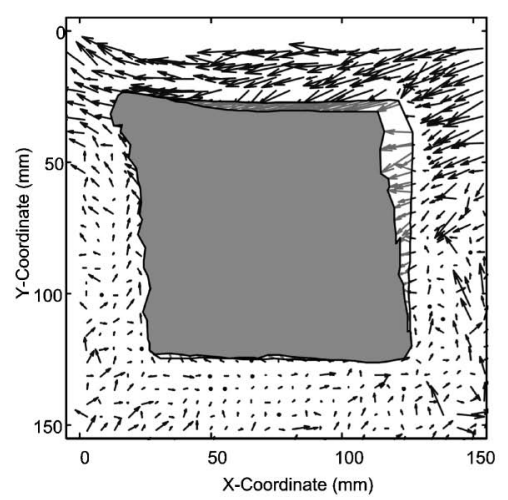

UC11

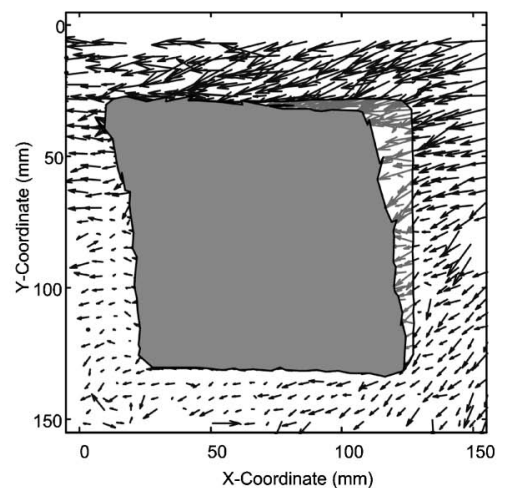

Fig. 9 Soil and tunnel deformations from zero to maximum acceleration phase measured with PIV technique (model scale). Deformations are magnified by 100

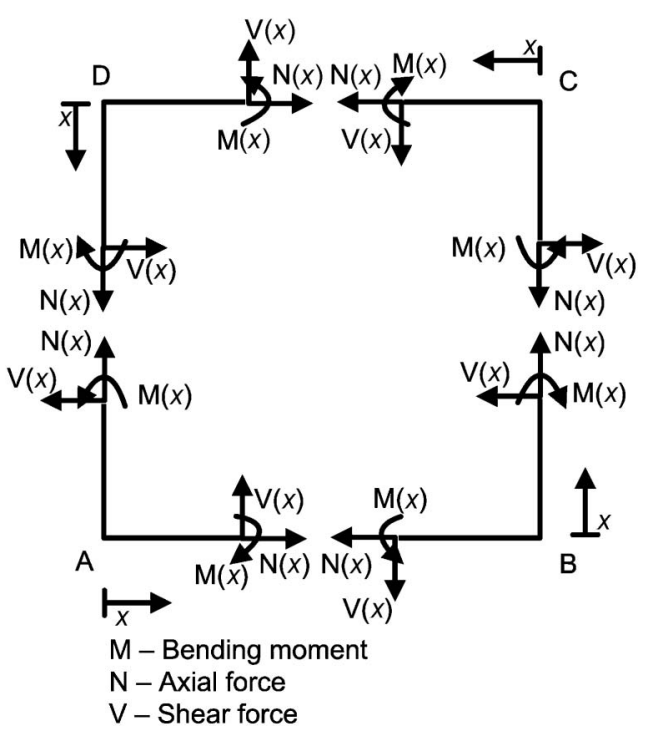

Fig. 10 Sign convention for the lining forces

but only changes the magnitude of the dynamic bending moments.

On the other hand, dynamic bending moment distribution for models UC04 and UC06 ( $F=14$ and 20 respectively), shows that shallow tunnels experience larger bending moments compared to the deep tunnels (see Fig. 12). The relationship between cyclic, maximum and residual bending moments is similar to that in relatively flexible tunnels. Cyclic bending moments are more evenly distributed compared to the UC10 and UC11 $\left(F_{\text {square }}=330\right.$ and 247 respectively), which shows that the relatively rigid lining resists to the deformations more than the flexible lining; bending moments are distributed, because the corners resist the formation of plastic hinges.

Residual deformations of the four model tunnels predicted FEA is shown in Fig. 13. They are found by comparing the initial tunnel configuration with the configuration at the end of the earthquake. The deformations are all scaled by magnification factor of 250 to enhance the deformation patterns. The difference between
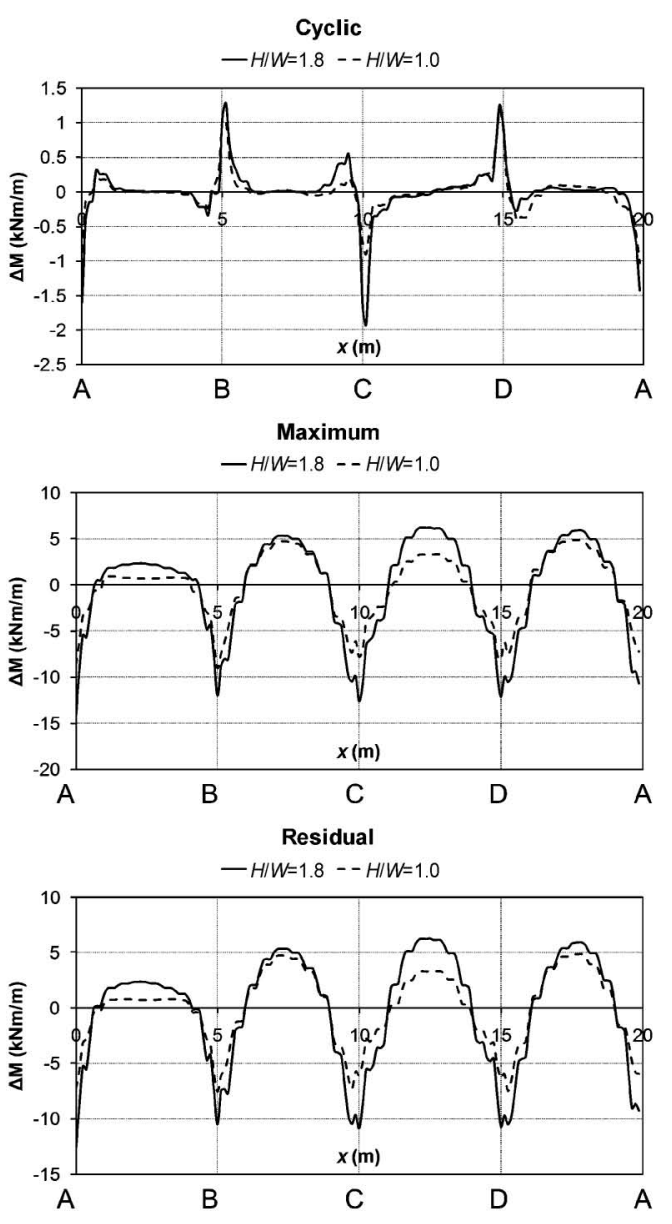

Fig. 11 Dynamic bending moments in relatively flexible tunnel linings $(\mathrm{UC10}-\mathrm{H} / \mathrm{W}=1.8$ and $\mathrm{UC11}-\mathrm{H} / \mathrm{W}=1.0)$ at prototype scale

the magnitude of deformation between relatively flexible and relatively rigid tunnels is apparent. However, the difference between deep and shallow rigid tunnels is small. For relatively rigid tunnels, shallower the tunnel axis, larger the deformations are. Opposite is true for the relatively flexible tunnels i.e. shallower the tunnel axis, smaller the deformations. Overall, shallow flexible tunnels deform less than the deep flexible tunnels. 

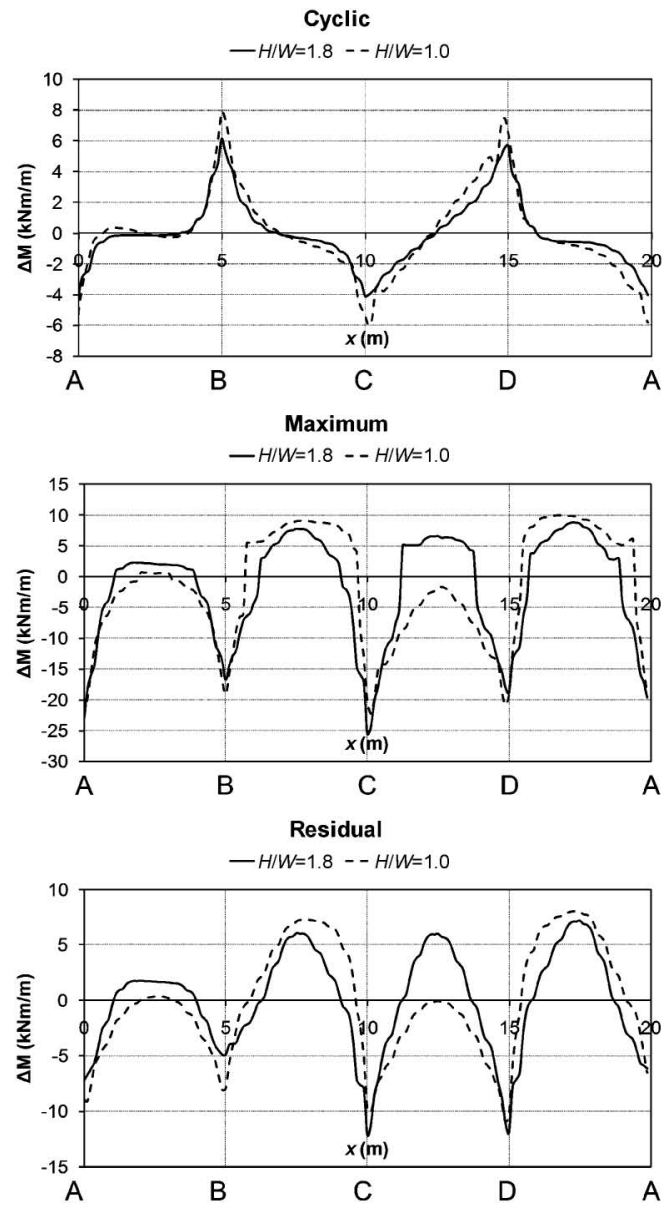

Fig. 12 Dynamic bending moments in relatively rigid tunnel linings (UC04-H/W $=1.0$ and $\mathrm{UC06}-\mathrm{H} / \mathrm{W}=1.8$ ) at prototype scale
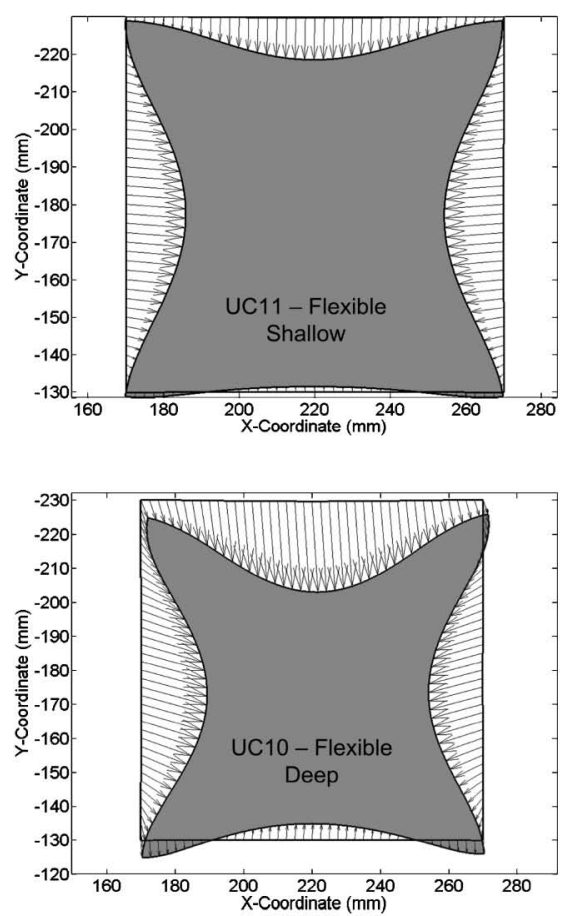

\section{CONCLUSIONS}

Seismic behaviour of shallow tunnels in a dry sand deposit was investigated using dynamic centrifuge modelling and complimentary Finite Element analyses. The deformation of the model tunnels in centrifuge tests were obtained by applying PIV techniques to high resolution images obtained from a high speed camera. Results have shown that the dynamic response of square tunnels can be split into three main stages: initial transient stage, subsequent steady-state cycles and finally a residual static stage. First couple of cycles defines the initial stage where most of the plastic deformations occur. Then follows the steady-state cycles where accumulation of the plastic deformations is low compared to what tunnel experiences in initial stage and the tunnel does racking movement until the end of the earthquake where the tunnel enters into static stage, where residual earth pressures and lining forces are left on the lining. It has been shown that the seismic behaviour of the tunnel cannot be simplified by just using sway type analysis. A critical mode of deformation is the deformation of the crown, invert and side walls as a result of plastic strains developing in the initial stages of strong shaking. For the cases investigated in this paper, the depth of the tunnel axis is less significant with regards to the mechanism of deformation; however depth of tunnel axis changes the amount of amplification of accelerations through the tunnel, the magnitude of dynamic earth pressures and the magnitude of the lining forces. Following conclusions can be drawn regarding the effect of depth on the seismic response of the square tunnels:

- Amplification of the accelerations from bedrock to the top of the tunnel is affected by the depth of the
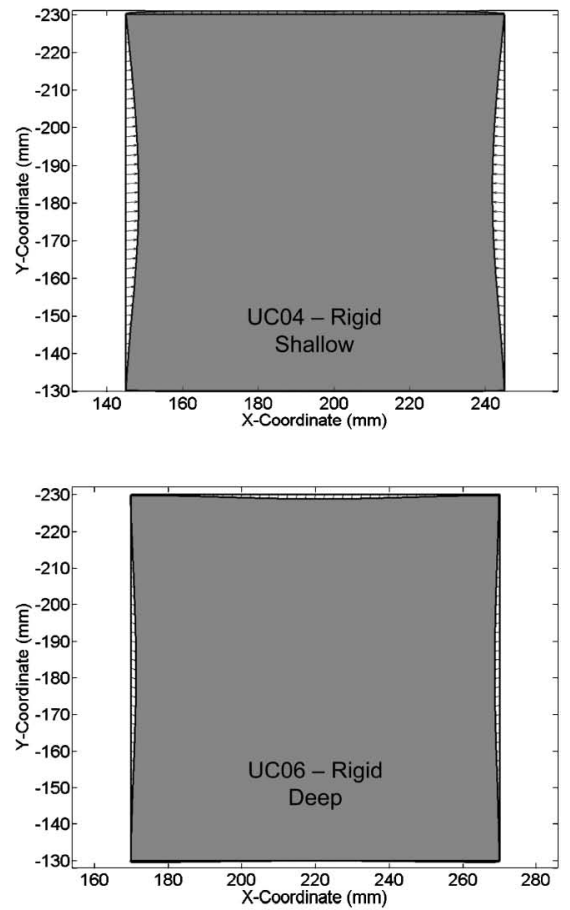

Fig. 13 Residual lining deformations predicted by FEA. Arrows are scaled by 250 
tunnel. Larger amplifications were observed for shallow model compared to the deep model for whole range of frequencies. For frequencies higher than 6 $\mathrm{Hz}$ the difference between amplification values gets bigger.

- Peak acceleration above the tunnel gets larger than the acceleration in free-field as depth-to-width ratio increases.

- FE results and centrifuge measurements reveal that deeper tunnels exhibit larger changes in dynamic earth pressure values. Maximum changes occur near the corners of the tunnel.

- PIV analyses do not show substantial difference between the magnitude of deformations in shallow and deep tunnel models. However, for deep tunnel model, crown of the tunnel deforms more than the crown of shallow tunnel model.

- Cyclic, maximum and residual dynamic bending moments in flexible deep tunnel model are higher compared to the flexible shallow tunnel model. Converse is true for rigid tunnels. Dynamic bending moments are smaller for rigid deep tunnel compared to rigid shallow tunnel.

It should be noted that these conclusions were drawn from limited number of tests. The tests reported herein only include flexible tunnels with shallow embedment depths. More experimental and field data is needed in order to set up a framework for the effect of depth on the mechanisms related to the seismic behaviour of tunnels.

\section{ACKNOWLEDGEMENTS}

Authors are grateful for the financial support received from Yuksel Proje Uluslararasi for this research. Excellent technical support from technicians at the Schofield Centrifuge Centre, Cambridge (UK) is also greatly acknowledged.

\section{NOTATION}

$H$-Height of the tunnel

$W$-Width of the tunnel

$G_{\mathrm{m}}$-Shear modulus of the soil

I-Moment of inertia of the lining (per unit width)

$E_{1}-$ Young's modulus of the lining material

$\zeta_{\mathrm{i}}$-Damping ratio at mode $\mathrm{i}$

$\omega_{\mathrm{i}}-$ Circular frequency of the mode $\mathrm{i}$

$\alpha_{\mathrm{R}}$-Mass proportional Rayleigh damping coefficient

$\beta_{\mathrm{R}}$-Stiffness proportional Rayleigh damping coefficient

$\Delta t$-Time Increment

\section{REFERENCES}

1) Brennan, A. J. (2003): Vertical drains as a countermeasure to earthquake-induced soil liquefaction, Ph.D. thesis, Cambridge Univer- sity.

2) Campbell, D. J., Cheney, J. A. and Kutter, B. L. (1991): Boundary effects in dynamic centrifuge model tests, Proc. Centrifuge 91, Balkema, Rotterdam, 441-448.

3) Cheney, J. A., Hor, O. Y. Z., Brown, R. K. and Dhat, N. R. (1988): Foundation vibration in centrifuge models, Proc. Centrifuge 88, Balkema, Rotterdam, 481-486.

4) Ghosh, B. and Madabhushi, S. P. G. (2002): An efficient tool for measuring shear wave velocity in the centrifuge, Proc. International Conference on Physical Modelling in Geotechnics, Balkema, 1, 119-124.

5) Haigh, S. K., Ghosh, B. and Madabhushi, S. P. G. (2005): Importance of time step discretisation for nonlinear dynamic finite element analysis, Canadian Geotechnical Journal, 42(3), 957-963.

6) Hardin, B. O. and Drnevich, V. P. (1972): Shear modulus and damping in soils: design equations and curves, Journal of the Soil Mechanics and Foundations Division, ASCE, 98(SM7), 667-692.

7) Hashash, Y. M. A., Hook, J. J., Schmidt, B. and Yao J. I.-C. (2001): Seismic design and analysis of underground structures, Tunneling and Underground Space Technology, 16, 247-293.

8) Kishida, H. and Uesugi, M. (1987): Tests of the interface between sand and steel in the simple shear apparatus, Géotechnique, 37(1), 45-52.

9) Lenke, L. R., Pak, R. Y. S. and Ko, H. Y. (1991): Boundary effects in modelling of foundations subjected to vertical excitation, Proc. Centrifuge 91, Balkema, Rotterdam, 473-480.

10) Madabhushi, S. P. G., Schofield, A. N. and Lesley, S. (1998): A new stored angular momentum (SAM) based earthquake actuator, Proc. of Centrifuge '98, Balkema, Rotterdam, 111-116.

11) Pakbaz, M. C. and Yareevand, A. (2005): 2-d analysis of circular tunnel against earthquake loading, Tunneling and Underground Space Technology, 20, 411-417.

12) Penzien, J. (2000): Seismically induced racking of tunnel linings, Earthquake Engineering and Structural Dynamics, 29, 683-691.

13) Schofield, A. N. (1981): Dynamic and earthquake geotechnical centrifuge modelling, Proc. of Int. Conf. on Recent Advances in Geotechnical Earthquake Engineering and Soil Dynamics, University of Missouri-Rolla, MO, USA, 1081-1100.

14) Shibayama, S., Izawa, J., Takahashi, A., Takemura, J. and Kusakabe, O. (2010): Observed behaviour of a tunnel in sand subjected to shear deformation in a centrifuge, Soils and Foundations, 50(2), 281-294.

15) Steedman, R. S. and Madabhushi, S. P. G. (1991): Wave propagation in sand medium, Proc. Intl. Conf on Seismic Zonation, Stanford University, Stanford, California.

16) Thusyanthan, I. (2005): Behaviour of landfill systems under monotonic and earthquake loading, PhD thesis, Department of Engineering, University of Cambridge.

17) Wang, J.-N. (1993): Seismic Design of Tunnels: A state-of-the-art approach, monograph, New York: Parsons, Brinckerhoff, Quade and Dougless Inc.

18) Wang, W. L., Wang, T. T., Su, J. J., Lin, C. H., Seng, C. R. and Huang, T. H. (2001): Assessment of damage in mountain tunnels due to the taiwan chi-chi earthquake, Tunneling and Underground Space Technology, 16, 133-150.

19) Wang, Z., Gao, B., Jiang, Y. and Yuan, S. (2009): Investigation and assessment on mountain tunnels and geotechnical damage after the wenchuan earthquake, Science in China Series E: Technological Sciences, 52(2), 546-558.

20) White, D. J. (2002): GeoPIV: Particle Image Velocimetry (PIV) software for use in geotechnical testing, University of Cambridge Department of Engineering, Technical Report, CUED/D-SOILS/ TR322. 